\title{
Incremento de la participación mediante la gamificación en la asignatura Dirección de Operaciones del Grado en Administración y Dirección de Empresas
}

\section{Increased participation through gamification in the course Operations Management of the Degree in Business Administration and Management}

\author{
DOI: $10.46932 / \mathrm{sfjdv2n3-073}$
}

Received in: May 1st, 2021

Accepted in: Jun 30th, 2021

\author{
Miguel Ángel Montañés Del Río \\ Profesor Doctor del DeparProfesor Doctor Departamento de Organización de Empresas de la \\ Universidad de Cádiz \\ Facultad de Ciencias Sociales y de la Comunicación, Universidad de Cádiz \\ Av. Universidad S/N (11405). Jerez de la Frontdera, Cádiz (España) \\ E-mail: miguelangel.montanes@uca.es
}

\author{
Vanessa María Rodríguez Cornejo \\ Profesor Doctor del DeparProfesora Doctora Departamento de Organización de Empresas de la \\ Universidad de Cádiz \\ Facultad de Ciencias Sociales y de la Comunicación, Universidad de Cádiz \\ Av. Universidad S/N (11405). Jerez de la Frontdera, Cádiz (España) \\ E-mail: vanesa.rodriguez@uca.es \\ Margarita Ruiz Rodríguez \\ Profesor Doctor del DeparProfesora Doctora Departamento de Organización de Empresas de la \\ Universidad de Cádiz \\ Facultad de Ciencias Sociales y de la Comunicación, Universidad de Cádiz \\ Av. Universidad S/N (11405). Jerez de la Frontdera, Cádiz (España) \\ E-mail:margarita.ruiz@uca.es

\section{Jaime Sánchez Ortiz} \\ Profesor Doctor del DeparProfesora Doctora Departamento de Organización de Empresas de la \\ Universidad de Cádiz \\ Facultad de Ciencias Sociales y de la Comunicación, Universidad de Cádiz \\ Av. Universidad S/N (11405). Jerez de la Frontdera, Cádiz (España) \\ E-mail: jaime.sanchez@uca.es
}

\section{RESUMEN}

En la actual sociedad de la información y del conocimiento se observa cómo la proliferación y el auge de los dispositivos móviles, hacen surgir nuevos modelos de aprendizaje que reformulan las metodologías didácticas, organizativas, y de formación de los currículos, para innovar en educación (Moreno Martínez, Leiva Olivencia, \& Matas Terrón, 2016). Los estudios que identifican las tecnologías emergentes que podrían impactar en el aprendizaje, la enseñanza y la investigación, prevén el aumento del uso y la incorporación de dispositivos y aplicaciones móviles en las aulas (Reig, 2013; Reig \& Vílchez, 2013). Destaca el aprendizaje basado en juegos o gamificación, que permite desarrollar procesos de enseñanzaaprendizaje que facilitan la cohesión, la integración y la motivación, potenciando la creatividad del alumnado. Crear juegos entretenidos motivará al alumnado para que participe de forma activa en clase, fomentará una sana competencia entre los jugadores, y hará del aprendizaje algo más divertido (Aleksić- 
Maslać, Rašić, \& Vranešić, 2018). Utilizando Kahoot en dispositivos móviles como soporte para las clases de Dirección de Operaciones del Grado en Administración y Dirección de Empresas de la Universidad de Cádiz, se consiguió motivar al alumnado y, aun existiendo otros factores determinantes, mejorar sus resultados de evaluación.

Palabras clave: Gamificación, Kahoot, Dirección de Operaciones, Motivación

\begin{abstract}
In today's information and knowledge society, the proliferation and rise of mobile devices has led to the emergence of new learning models that reformulate didactic, organizational and curricular training methodologies in order to innovate in education (Moreno Martínez, Leiva Olivencia, \& Matas Terrón, 2016). Studies that identify emerging technologies that could impact learning, teaching and research, foresee increased use and incorporation of mobile devices and applications in classrooms (Reig, 2013; Reig \& Vílchez, 2013). Learning based on games or gamification stands out, which allows the development of teaching-learning processes that facilitate cohesion, integration and motivation, boosting students' creativity. Creating entertaining games will motivate students to participate actively in class, encourage healthy competition between players, and make learning more fun (Aleksić-Maslać, Rašić, \& Vranešić, 2018). Using Kahootin mobile devices as support for the classes of Operations Management of the Degree in Business Administration and Management of the University of Cadiz, it was possible to motivate the students and, although there are other determining factors, to improve their evaluation results.
\end{abstract}

Keywords: Gamification, Kahoot, Operations Management, Motivation

\title{
1 INTRODUCCIÓN
}

El proceso educativo basado en las lecciones magistrales se ha visto influido por el rápido desarrollo de las nuevas tecnologías, modificándose los roles desempeñados por docentes y discentes (Aleksić-Maslać et al., 2018). En educación superior la pasiva respuesta del alumnado muestra la ineficiencia de este método (Larsen, 2006), que llega a ser tedioso para las nuevas generaciones, y convierte en un reto mantener al alumnado atento en clase durante toda la jornada lectiva (Aleksić-Maslać et al., 2018).

Así, el actual panorama educativo requiere incorporar los avances tecnológicos para permitir mejorar la calidad de la enseñanza y del proceso de aprendizaje (Molina Álvarez, Ortiz Colón, \& Agreda Montoro, 2017). Frente a los tradicionales métodos de aprendizaje que han demostrado falta de motivación en el alumnado (Simões, Redondo, \& Vilas, 2013), irrumpen los juegos como uno de los medios para poder motivar y atraer la atención de estas nuevas generaciones de estudiantes a través, entre otros factores, de la competición (Hanus \& Fox, 2015). Para Kenny y McDaniel (2011), los juegos fomentan la motivación del alumnado gracias a su componente lúdico, confirmándose la relación motivación-gamificación (Molina Álvarez et al., 2017).

Los juegos representan, por lo tanto, una gran oportunidad para evitar el abandono del alumnado, la desmotivación, la desgana, o la ausencia de compromiso en su proceso de enseñanza-aprendizaje 
(Mérida Serrano, Angulo Romero, Jurado Bello, \& Diz Pérez, 2011). Su incorporación en la educación, conocido por gamificación, persigue fomentar la motivación, el compromiso y determinados comportamientos (Lee \& Hammer, 2011).

Por otra parte, el creciente uso diario de recursos tecnológicos ha conducido a que terminen por implantarse también en las aulas, respondiendo así de forma efectiva a las demandas del alumnado (Curto Prieto, Orcos Palma, Blázquez Tobías, \& Molina León, 2019). En este sentido, la utilización de sistemas de respuesta personal (Student Response Systems) en las clases puede no solo estimular una mayor asistencia debido a su popularidad entre el alumnado (Castilla, Romana, \& López-Terradas, 2013), sino también aumentar el disfrute por un curso docente (Abrahamson, 1998).

La utilización en clase de la aplicación gratuita Kahoottrata de aumentar la motivación del alumnado y, en consecuencia, mejorar sus calificaciones (San-Miguel, Megías, \& Serna, 2017), por lo que comprobar si las calificaciones del alumnado en la asignatura Dirección de Operaciones II, impartida en el Grado en Administración y Dirección de Empresas de la Universidad de Cádiz durante el curso académico 2018-2019, han mejorado respecto a las del curso anterior a consecuencia de la mayor motivación de aquel por el uso en clase de cuestionarios realizados con Kahoot, es el principal objetivo de este artículo.

\section{CONTEXTO}

\subsection{GAMIFICACIÓN}

Las organizaciones, Universidades inclusive, se enfrentan a una crisis de compromiso debido al limitado tiempo del que disponen las personas que las integran. Para evitar perderlo, estas últimas crean escudos que tan solo unas pocas organizaciones atraviesan al generar cierta vinculación con ellas. En este sentido, la gamificación es una estrategia emergente (Burke, 2014).

La definición de gamificación es compleja y está poco consensuada (Molina Álvarez et al., 2017). De origen anglosajón, el término describe la utilización de técnicas, elementos y dinámicas que, propias de los juegos, se trasladan a otros ámbitos ajenos a los mismos (Jaber et al., 2016) para intentar transmitir unos contenidos que, por medio de una experiencia lúdica, potencien aspectos como la motivación, la implicación, el aprendizaje o la diversión (Molina Álvarez et al., 2017), pero también la concentración y el esfuerzo entre grupos de personas (Castilla et al., 2013).

Pero el uso de dinámicas y mecánicas de juego en entornos no lúdicos no es algo nuevo (Molina Álvarez et al., 2017). Desde hace tiempo se "acumulan millas" al volar con las aerolíneas lo que, unido a la aparición de dispositivos móviles y aplicaciones descargables, ha servido para popularizar su utilización en otros ámbitos (Smith-Robbins, 2011). 
El esfuerzo por acumular puntos y escalar categorías tras completar una serie de pruebas es extrapolable al sistema educativo, donde la titulación otorgada por una Institución acreditada sería no solo la categoría alcanzada sino también una muestra de distinción, y las calificaciones logradas en cada asignatura, las puntuaciones (Smith-Robbins, 2011). No obstante, y a nivel universitario, apenas se han llegado a plantear experiencias que vuelquen lo positivo del mecanismo de los juegos (Jaber et al., 2016).

Es mucho el alumnado que percibe la educación tradicional como algo aburrido y en ocasiones poco eficaz, lo que supone que el profesorado busque continuadamente nuevos métodos de enseñanza para motivarlo y captar su compromiso con las asignaturas (Contreras Espinosa \& Eguia, 2016). La motivación del alumnado en el aula constituye, por lo tanto, una de las mayores preocupaciones del docente (Castilla et al., 2013) porque, cuando se está motivado, se obtienen mejores resultados (Santana Cabello \& García Juan, 2018).

La gamificación incorpora estrategias para hacer clases más motivadoras y que el alumnado quiera aprender (de Soto García, 2018). Se aprende mejor cuando se está motivado (Bergin \& Reilly, 2005), y cuando el alumnado se divierte al realizar una tarea, la información que recibe se fija en su cerebro generando mejores aprendizajes (Molina Álvarez et al., 2017).

Pero la gamificación no consiste en un juego en sí mismo sino que, antes bien, implica utilizar los elementos básicos que lo componen en contextos o entornos que no son lúdicos, como en la enseñanza, introduciendo en el aula recompensas, insignias, puntuaciones y dinámicas competitivas (Simões et al., 2013). Las principales definiciones de gamificación, de hecho, subrayan que no se trata tanto de crear un juego como de incorporar en otras actividades aquello que lo hace tan atractivo, siendo por ello que la teoría de la gamificación supone que el diseño apropiado de un juego puede cambiar el comportamiento humano y aumentar su productividad (Jakubowski, 2014).

En consecuencia, la gamificación es otra actividad de aprendizaje adicional cuyos fines pedagógicos trascienden el objetivo de la motivación (Molina Álvarez et al., 2017). Por lo tanto, los juegos adaptados al ámbito educativo deberían contar con dinámicas centradas en retos, recompensas, etc., que hicieran las clases más atractivas para el alumnado y que, además, pudiesen mejorar sus resultados académicos al fomentar una participación más activa en clase (de Soto García, 2018).

Para Díaz Cruzado y Troyano Rodríguez (2013), dos son los grupos en que se suelen dividir las actividades gamificadas: (1) juegos educativos convencionales que no precisan de un soporte electrónico, y; (2) actividades con soporte digital que, a diferencia de los anteriores, permiten incluir imágenes, sonidos, vídeos, etc., y que conforman un espacio de juego más atractivo, siendo Kahooty Socrative las aplicaciones de este tipo más usadas en las aulas. 


\subsection{VENTAJAS E INCONVENIENTES DE LA GAMIFICACIÓN}

Algunos beneficios derivados de utilizar actividades gamificadas en el aula son (Kay \& LeSage, 2009): (1) que al generar mayor interacción entre iguales para discutir posibles soluciones y elegir la más apropiada, se mejora el proceso de enseñanza-aprendizaje; (2) que al aumentar la atención del alumnado, más concentrado en lo que debe hacer en clase, y participando de forma activa al competir con otros compañeros, se mejora el ambiente, y; (3) que al obtenerse una retroalimentación instantánea sobre los resultados, mejora el sistema de evaluación del alumnado.

Las opiniones contrarias a la gamificación se basan, por un lado, en la idea de que la simple suma de puntos no hará que las personas quieran, de forma automática, obtenerlos. Y, por otro lado, en el hecho de que permitir que una persona pueda conseguir más puntos que otra no ha de servirle, necesariamente, de motivación (Smith-Robbins, 2011). La gamificación, por otra parte, puede hacer ver al alumnado que solo debería aprender cuando se le facilitaran recompensas externas (Lee \& Hammer, 2011).

\subsection{KAHOOTCOMO GAME-BASED STUDENT RESPONSE SYSTEM}

Kahoot, como integrante de los Sistemas de Respuesta del Alumnado Basados en el Juego (GSRS por su acrónimo inglés), es una popular y gratuita herramienta de eLearning, fácil de aprender y de usar, que consigue la participación y compromiso del alumnado, añadiendo vitalidad y apoyos meta-cognitivos (retroalimentación instantánea, p.e.) a las clases de educación superior con muy poco esfuerzo del profesorado (Plump \& LaRosa, 2017).

El profesorado lanza desde su ordenador el juego, que se proyecta sobre una pantalla gigante, y su alumnado se conecta mediante un dispositivo móvil a una página web (https://kahoot.it/) desde la que introduce un código (actualmente, un PIN de seis dígitos) que le permitirá participar en el cuestionario (Parra Santos, Molina Jordá, \& Casanova Pastor, 2018). Al no ser preciso tener que registrarse, ni facilitar su auténtico nombre, el alumnado puede concursar de forma anónima y, en consecuencia, sentirse menos presionado por los fallos.

Al terminar la actividad, Kahoot, facilita automáticamente los resultados obtenidos, por lo que se pueden ver los aciertos y los errores cometidos para establecer un período de debate que, por lo general, suele ser bien recibido por el alumnado al no apreciarlo como una crítica de su trabajo por estar en un ambiente lúdico (de Soto García, 2018).

Se decidió utilizar Kahoot, porque la inclusión de cuestionarios tipo test facilita el aprendizaje y la memoria (Toppino \& Gerbier, 2014), ya que permiten comprobar al alumnado lo que sabe y lo que no, concentrando sus esfuerzos en el estudio de aquellas áreas con menor conocimiento (Roediger III, Putnam, \& Smith, 2011). 


\subsection{VENTAJAS E INCONVENIENTES DE KAHOOT}

La atención es un elemento de motivación y un prerrequisito para el aprendizaje (Keller, 1987), luego conseguirla del estudiante es una cuestión fundamental para su aprendizaje (Gagne \& Driscoll, 1988), y Kahootlo hace.

Además de cuestionarios, se pueden realizar rompecabezas, gráficos y discusiones, lo que refuerza el aprendizaje (Dellos, 2015). También, y como las respuestas se muestran en la pantalla con distintos colores, y con una melodía que recuerda a los videojuegos, su formato atrae al alumnado (de Soto García, 2018).

Tabla 1. Ventajas de utilizar Kahoot en el aula

\begin{tabular}{|l|}
\hline Gratuito \\
\hline Fácil de aprender para el profesorado \\
\hline $\begin{array}{l}\text { Procesos simples para el alumnado (no precisa una cuenta } \\
\text { para registrarse ni descargar aplicación alguna) }\end{array}$ \\
\hline $\begin{array}{l}\text { Compatible con smartphones, tabletas (dispositivos } \\
\text { electrónicos móviles) u ordenadores personales }\end{array}$ \\
\hline $\begin{array}{l}\text { La obtención de resultados en tiempo real permite al } \\
\text { profesorado facilitar aclaraciones sobre contenidos allí } \\
\text { donde sean precisas }\end{array}$ \\
\hline La música y los colores animan al estudiante \\
\hline $\begin{array}{l}\text { Aumenta el compromiso del alumnado al ser atraído por sus } \\
\text { colores, la música y la excitación que la competencia genera }\end{array}$ \\
\hline $\begin{array}{l}\text { El profesorado puede descargar, revisar y guardar los } \\
\text { resultados del alumnado }\end{array}$ \\
\hline $\begin{array}{l}\text { El alumnado puede intentar los cuestionarios cuantas veces } \\
\text { quiera }\end{array}$ \\
\hline $\begin{array}{l}\text { El profesorado puede crear cuestionarios, preguntas de } \\
\text { discusión o encuestas }\end{array}$ \\
\hline $\begin{array}{l}\text { El profesorado puede ajustar, según convenga, el tiempo } \\
\text { permitido al alumnado para responder }\end{array}$ \\
\hline $\begin{array}{l}\text { La participación en los cuestionarios sin la necesidad de } \\
\text { facilitar el nombre real, motiva que la clase se implique en } \\
\text { su realización }\end{array}$ \\
\hline
\end{tabular}

Fuente: Adaptado de Plump y LaRosa (2017)

Entre las limitaciones de Kahoot, están el tiempo requerido para su preparación y puesta en práctica, la necesidad de tener conexión permanente a Internet, la posibilidad de que el alumnado copie sus respuestas (Martínez Navarro, 2017), el que no se pueda marcar más de una respuesta como correcta, la limitación en el número de caracteres incluidos en las respuestas, o una excesiva competitividad. Esto último podría generar efectos adversos, y siempre sería posible contar con un porcentaje de clase desmotivada por haberse clasificado en las posiciones más bajas (de Soto García, 2018).

\section{DESCRIPCIÓN}




\subsection{INFORMACIÓN PREVIA DE LA ASIGNATURA}

La Dirección de Operaciones impartida en la Universidad de Cádiz (en lo sucesivo, UCA) por el Departamento de Organización de Empresas, se distribuye en dos cursos sucesivos del Grado en Administración y Dirección de Empresas (en adelante, ADE). En ellos se muestran, respectivamente, los aspectos estratégicos (Dirección de Operaciones I) y los operativos (Dirección de Operaciones II).

La investigación aquí realizada se refiere a Dirección de Operaciones II (en lo sucesivo, DOPII), con una carga docente de 6 ECTS que se distribuyen en teoría (4 ECTS) y práctica (2 ECTS), y se imparte con carácter obligatorio en el primer semestre del cuarto curso del plan de estudios del Grado en ADE en tres de los Campus de la UCA: Cádiz, Jerez de la Frontera y Algeciras. La evaluación de esta asignatura considera los siguientes ítems:

- Cuestionarios tipo test de respuesta múltiple y solo una correcta. Para un total de siete de ellos, el peso de este ítem sobre la nota final es de un $10 \%$.

- Dos ejercicios prácticos individuales a realizar en clase. Su peso conjunto sobre la nota final de la asignatura es igualmente del $10 \%$.

- Examen presencial. Su peso es del $80 \%$ en la nota final, y abarca dos partes: una teórica y otra práctica. En ambas se debe conseguir una puntuación mínima del 30\% de la nota otorgada a las mismas, lo que también se aplica para cada uno de los tres ejercicios prácticos propuestos. Además de lo anterior, y en su caso, la suma de la nota obtenida en cada una de dichas partes deberá alcanzar el $50 \%$ de la nota otorgada al examen presencial.

La fijación de una nota mínima de corte para aprobar supone, a la vista de la experiencia docente ya acumulada, cierta frustración entre el alumnado. Por ello, se planteó un Proyecto de Innovación y Mejora Docente (en adelante PIMD) basado en la utilización en el aula de herramientas didácticas alternativas como Kahoot, con el que se pretendió estudiar si era posible revertir la situación, motivar al alumnado, y que obtuviera mejores calificaciones.

\subsection{DISEÑO DE LA INVESTIGACIÓN}

El estudio realizado tomó una muestra de 60 estudiantes de la asignatura DOPII en los Campus de Jerez de la Frontera y de Algeciras durante el primer semestre del curso académico 2018/2019. El Campus de Cádiz, donde también hay docencia en DOPII, no participó en el estudio al haberse reducido drásticamente el alumnado asistente a clase.

Se confeccionaron siete cuestionarios de respuesta múltiple con Kahoot, uno por cada tema de la programación de DOPII y en la clase inmediatamente posterior a la terminación de cada tema, se solicitaba del alumnado asistente la utilización de sus dispositivos móviles para conectarse a los mismos. 
Al finalizar cada pregunta, y a modo de Flipped Classroom (Tucker, 2012), metodología de enseñanza basada en el aprendizaje activo utilizada, por ejemplo, en la asignatura Dirección de Operaciones impartida en la Universidad Rey Juan Carlos de Madrid (Díaz-Garrido, Martín-Peña, \& Sánchez-López, 2017), se establecía un breve debate con el alumnado para afianzar los conceptos mostrados en cada una de aquellas y determinar, mediante aprendizaje colaborativo entre los asistentes, el porqué de las respuestas incorrectas.

Además, y en la última de las clases destinadas a la docencia en DOPII, se le entregó al alumnado asistente un cuestionario de opinión final para conocer su grado de motivación con la asignatura, y su nivel de satisfacción con el PIMD ejecutado durante el curso 2018-2019. Se había de responder anónima y voluntariamente al objeto de conseguir mayor objetividad, estando su confección basada, previa adaptación al contexto particular de la investigación, en dos trabajos previos: (1) la encuesta de motivación con el curso realizada por Kebritchi, Hirumi y Bai (2010), y; (2) la investigación de San-Miguel, Megías y Serna (2017).

El cuestionario utilizó una escala Likert de 5 puntos sobre un total de 12 ítems. Los ítems del cuestionario final de opinión del alumnado, relacionados con su motivación y satisfacción, se recogen en la

tabla 2. Este tipo de escala suministra una base común para las respuestas a los ítems relacionados con diferentes aspectos de la experiencia universitaria (Douglas, Douglas, \& Barnes, 2006). Cuando se otorgaba 1 punto significaba estar "Nada de acuerdo", mientras que la valoración con 2 puntos suponía "Estar de acuerdo" y el valor neutro implicaba conceder 3 puntos. Los valores más altos otorgados en cada ítem, 4 y 5, suponían "Estar muy de acuerdo" y "Estar totalmente de acuerdo", respectivamente.

Tabla 2. Ítems motivación-satisfacción del cuestionario de opinión final en DOPII

\begin{tabular}{|l|l|}
\hline Ítem & Codificación \\
\hline $\begin{array}{l}\text { 3. Conocer la respuesta correcta justo } \\
\text { después de cada pregunta le ha ayudado } \\
\text { en su aprendizaje. }\end{array}$ & FDBCK \\
\hline $\begin{array}{l}\text { 4. La realización de este tipo de } \\
\text { cuestionarios ayuda en la asimilación de } \\
\text { los contenidos. }\end{array}$ & ASIM_CTDOS \\
\hline $\begin{array}{l}\text { 5. El debate realizado después de cada } \\
\text { pregunta del cuestionario permite } \\
\text { entender mejor muchos conceptos. }\end{array}$ & DEBT \\
\hline $\begin{array}{l}\text { 6. La realización de este tipo de actividad } \\
\text { permite seguir mejor la asignatura. }\end{array}$ & SEG_DOPII \\
\hline $\begin{array}{l}\text { 7. Usted prefiere realizar este tipo de } \\
\text { cuestionarios frente a los que se realizan } \\
\text { en pape y son entregados al profesor para } \\
\text { su posterior evaluación. }\end{array}$ & PRFRCIA_1 \\
\hline $\begin{array}{l}\text { 8. Usted prefiere realizar este tipo de } \\
\text { cuestionarios frente a los que se realizan } \\
\text { a través del aula virtual de la asignatura. }\end{array}$ & PRFRCIA_2 \\
\hline $\begin{array}{l}\text { 9. Le parece un sistema de evaluación } \\
\text { continuada original. }\end{array}$ & EVA_ORIG \\
\hline
\end{tabular}




\begin{tabular}{|l|l|l|}
\hline Ítem & Codificación \\
\hline $\begin{array}{l}10 . \quad \text { Le parece un sistema de } \\
\text { evaluación continuada muy útil. }\end{array}$ & EVA_UTIL \\
\hline $11 . \quad$ Le gustaría que este tipo de & OT_ASIGN \\
cuestionarios fuera incluido en otras & \\
asignaturas. & \\
\hline $12 . \quad$ Los elementos de innovación y & COMPR_CPTCIAS \\
mejora docente aplicados en esta & \\
asignatura han favorecido su \\
comprensión de los contenidos y/o la \\
adquisición de competencias asociadas a \\
la misma.
\end{tabular}

\section{RESULTADOS}

\subsection{CUESTIONARIO DE OPINIÓN FINAL}

En la

figura 1 se muestra, para cada ítem representativo de la motivación y de la satisfacción del alumnado en los Campus de Jerez de la Frontera y de Algeciras, la puntuación media otorgada al mismo por el alumnado que cursó DOPII. La media global de dichos ítems fue de 3,718 y la media de la desviación estándar fue de 1,188.

Figura 1. Motivación y satisfacción del alumnado tras utilizar Kahoot en DOPII

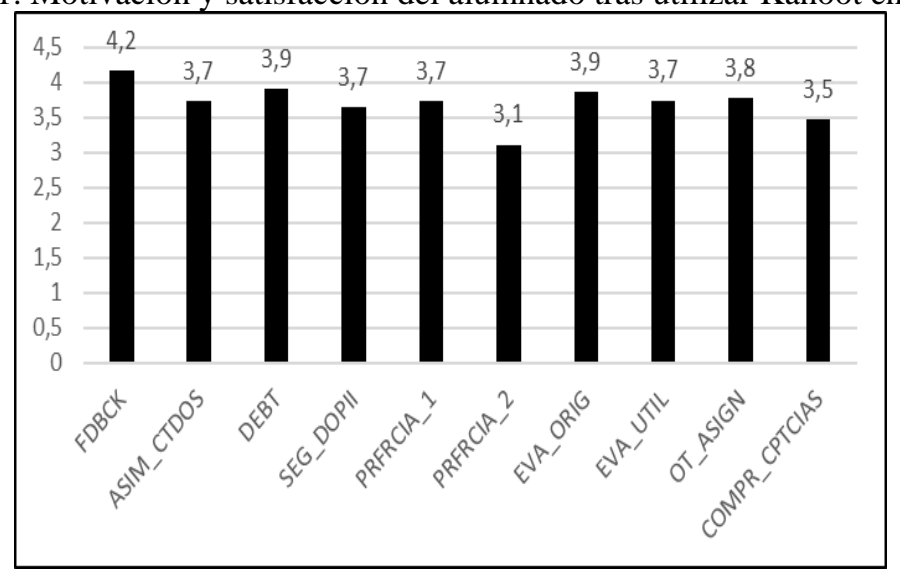

\subsection{CALIFICACIONES OBTENIDAS EN EL CAMPUS DE JEREZ DE LA FRONTERA}

Tomando como referencia las convocatorias de examen de febrero de 2018 y de febrero de 2019, el alumnado NO PRESENTADO a examen descendió un 25,64\%. Aun la existencia de otros factores explicativos, este dato se antoja un inequívoco síntoma de la motivación del alumnado por la asignatura tras la introducción de Kahoot en la docencia, bien por su orientación al resultado, bien por su orientación a la evitación (Alonso Tapia, 2007). 
La mayor participación provocó (véanse la figura 2 y la figura 3) un repunte del 18,18\% en el alumnado SUSPENSO que fue contrarrestado, sin duda alguna, por el espectacular incremento de quienes consiguieron aprobar la asignatura (el 35,29\% del alumnado presentado a examen).

La calificación de APROBADO aumentó un 29,41\%, pero destacó el crecimiento un 166,67\% del alumnado que obtuvo NOTABLE. Así, la nota media en la convocatoria de 2019 fue de 4,4875 suponiendo un aumento de poco más del 3\% respecto a la convocatoria de 2018.

Figura 2. Calificación cualitativa acta DOPII febrero 2018 del Campus de Jerez

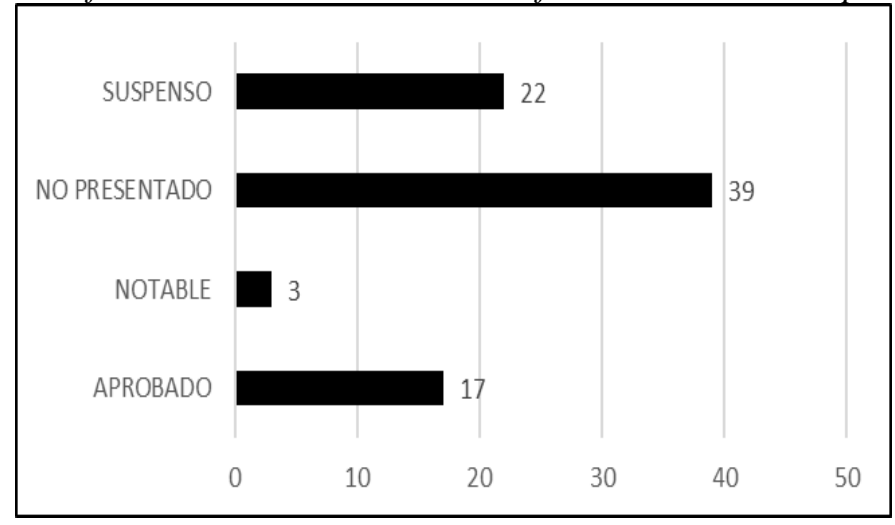

Figura 3. Calificación cualitativa acta DOPII febrero 2019 del Campus de Jerez

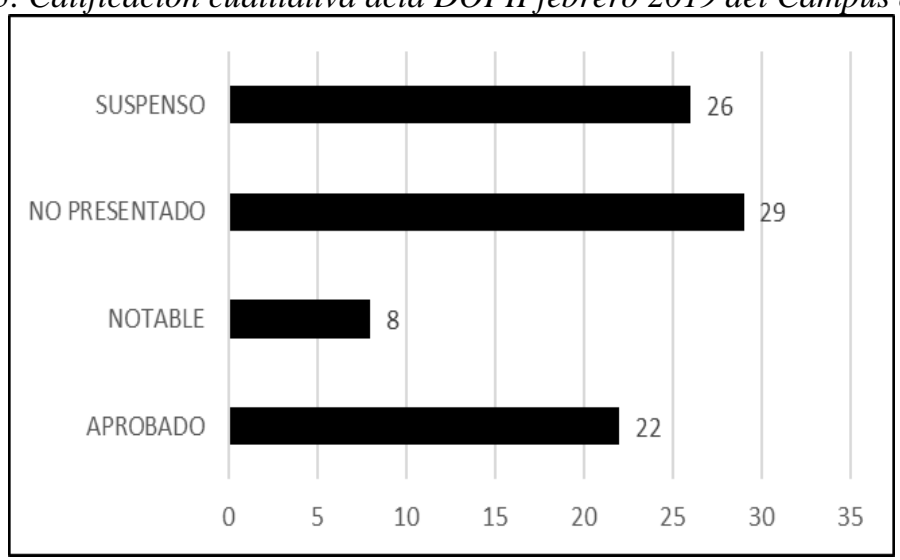

\section{CALIFICACIONES OBTENIDAS EN EL CAMPUS DE ALGECIRAS}

La nota media de DOPII en el Campus de Algeciras para la convocatoria de febrero de 2019 fue de 5,065 mientras que en la convocatoria de un año antes había sido un 5,83\% inferior. Es destacable, sin embargo, el acusado descenso del alumnado que figura en acta, cercano al $72 \%$.

De igual modo que ocurrió en el Campus de Jerez de la Frontera, y con respecto a la misma convocatoria de un año antes, el alumnado que dejó de presentarse a examen descendió en términos absolutos un 14,29\%, pero no en términos relativos a consecuencia de la caída en el número de estudiantes que figuraban en acta. 
El alumnado SUSPENSO también descendió en términos absolutos un 44,44\% (véanse la figura 4 y la figura 5) al igual que el alumnado con APROBADO, NOTABLE y SOBRESALIENTE (22,22\%, $7,69 \%$ y $100 \%$, respectivamente), lo que en términos absolutos se corrobora mediante una caída en el total de aprobados igual al 17,65\% con respecto a febrero de 2018. Esta caída, no obstante, estaría compensada con el incremento del alumnado con MATRÍCULA DE HONOR. En términos porcentuales, sin embargo, quienes aprueban DOPII en el Campus de Algeciras en la convocatoria de febrero de 2019 (45,90\%) superan a los que hicieron lo propio en la convocatoria de febrero de 2018 (41,46\%), lo que se podría explicar por el descenso del alumnado que figura en acta (de 82 se pasa a 61).

Figura 4. Calificación cualitativa acta DOPII febrero 2019 del Campus de Algeciras

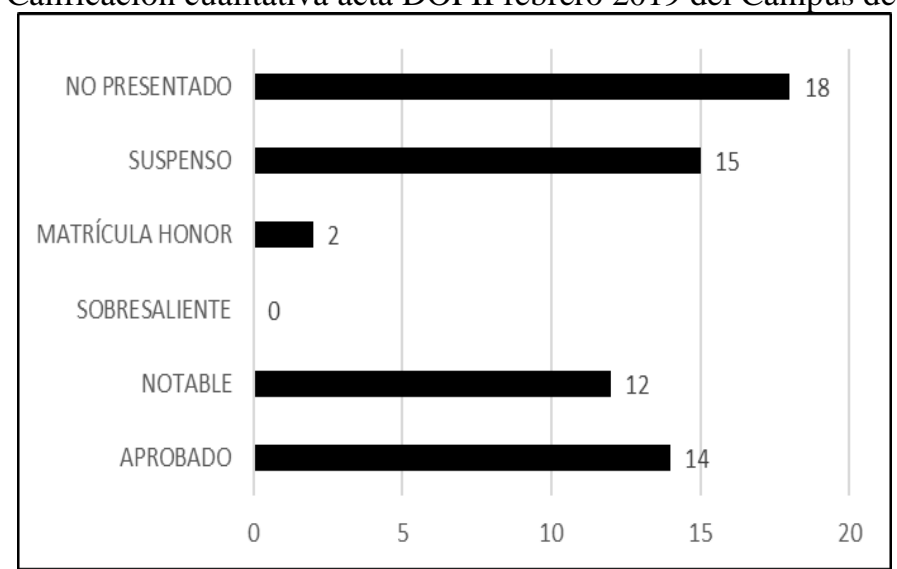

Figura 5. Calificación cualitativa acta DOPII febrero 2018 del Campus de Algeciras

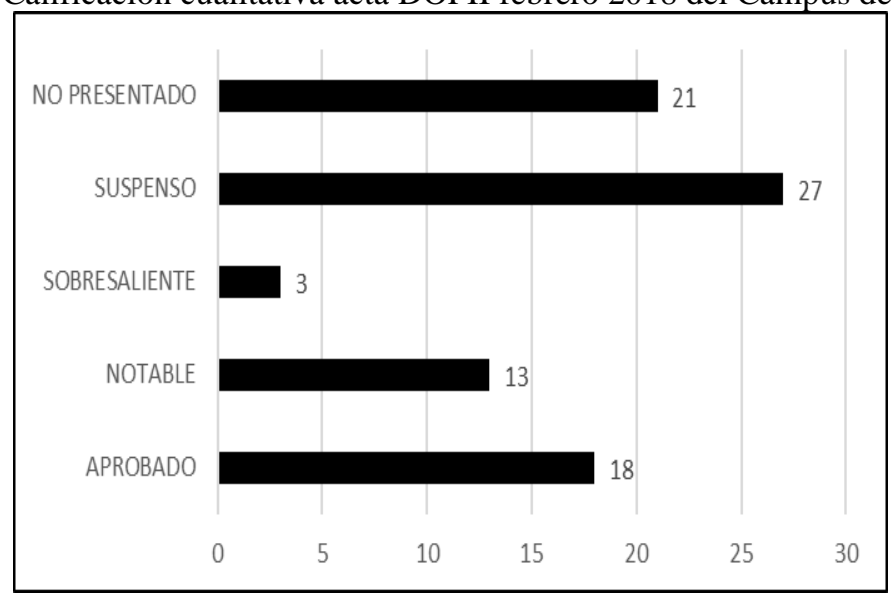

\section{CONCLUSIONES}

Los juegos pueden integrarse en una clase tradicional de educación superior para mejorar el aprendizaje, la motivación y el compromiso (Wang \& Lieberoth, 2016), demostrándose cómo mejoran los resultados académicos mediante procesos de enseñanza-aprendizaje gamificados (Molina Álvarez et al., 2017) y, concretamente a través de herramientas pedagógicas como Kahoot, observarse el potencial 
para obtener mejores calificaciones en los exámenes universitarios (Iwamoto, Taitano, Hargis, \& Vuong, 2017).

La gamificación ha sido una tendencia desde 2017, vaticinada por el NMC Horizon Report de 2014, materializada en juegos de simulación, desafíos y recompensas mediante puntos según el éxito alcanzado (Johnson, Adams Becker, Estrada, \& Freeman, 2014).

Dado que el alumnado que se matricula en DOPII ha expresado la complejidad que entraña el aprendizaje de sus materias y procedimientos, la elección de juegos instructivos podría ser más efectiva porque: (1) se actúa, no se explica; (2) se crea motivación personal y satisfacción entre el alumnado; (3) permite que se acomoden múltiples habilidades y estilos de aprendizaje; (4) refuerza las habilidades clave, y finalmente; (5) facilita un contexto interactivo para la toma de decisiones (Kebritchi et al., 2010).

Como lo que se pretende, entre otras cosas, es conseguir mayor motivación del alumnado y tratar de influir en su comportamiento para que adquiera nuevos contenidos por sí mismo, parece apropiado utilizar la gamificación en el ámbito educativo (Kapp, Blair, \& Rich, 2014).

La utilización de Kahooten el aula durante el curso 2018/2019 ha contribuido, junto a otros factores, al aumento de la motivación en el alumnado y, consecuentemente, a mejorar sus resultados académicos en DOPII respecto de la primera convocatoria del curso anterior. 


\section{REFERENCIAS}

Abrahamson, A. L. (1998). An overview of teaching and learning research with classroom communication systems. Samos International Conference on the Teaching of Mathematics. Samos, Greece.

Aleksić-Maslać, K., Rašić, M., \& Vranešić, P. (2018). Influence of gamification on student motivation in the educational process in courses of different fields. MIPRO 2018: 41st International Convention on Information and Communication Technology, Electronics and Microelectronics. Opatija, Croatia.

Alonso Tapia, J. (2007). Evaluación de la motivación en entornos educativos. In M. Á. y R. Bisquerra (Ed.), Manual de orientación y tutoría. Barcelona, España: Wolters Kluwer.

Bergin, S., \& Reilly, R. (2005). The influence of motivation and comfort-level on learning to program. In E. A. C. \& S. B. P. Romero, J. Good (Ed.), 17th Workshop of the Psychology of Programming Interest Group.

Burke, B. (2014). Gamify. How Gamification Motivates People to Do Extraordinary Things. https://doi.org/10.4324/9781315230344

Castilla, G., Romana, M. G., \& López-Terradas, B. (2013). Concursando en el aula: la gamificación mediante quizz-show como herramienta de dinamización docente. X Jornadas Internacionales de INnovación Universitaria: Educar Para Transformar. Madrid, España.

Contreras Espinosa, R. S., \& Eguia, J. L. (2016). Gamificación en las aulas universitarias (R. S. Contreras Espinosa \& J. L. Eguia, eds.). Institut de la Comunicació, Universitat Autónoma de Barcelona.

Curto Prieto, M., Orcos Palma, L., Blázquez Tobías, P., \& Molina León, F. J. (2019). Student Assessment of the Use of Kahoot in the Learning Process of Science and Mathematics. Education Sciences, 9(1). Retrieved from https://www.mdpi.com/2227-7102/9/1/55

de Soto García, I. S. (2018). Herramientas de gamificación para el aprendizaje de Ciencias de la Tierra. EDUTEC. Revista Electrónica de Tecnología Educativa, (65), 29-39. https://doi.org/dx.doi.org/10.21556/edutec.2018.65.1143

Dellos, R. (2015). KahootA digital game resource for learning. International Journal of Instructional Technology and Distance Learning, 12(4), 49-52. Retrieved from www.itdl.org/Journal/apr_15/apr15.pdf

Díaz-Garrido, E., Martín-Peña, M. L., \& Sánchez-López, J. M. (2017). The impact of Flipped Classroom on the motivation and learning of students in Operations Management. Working Papers on Operations Management, 8, 15. https://doi.org/10.4995/wpom.v8i0.7091

Díaz Cruzado, J., \& Troyano Rodríguez, Y. (2013). El potencial de la gamificación aplicado al ámbito educativo. III Jornadas de Innovación Docente. Innovación Educativa: Respuesta En Tiempos de Incertidumbre. Retrieved from https://idus.us.es/xmlui/handle/11441/59067

Douglas, J., Douglas, A., \& Barnes, B. (2006). Measuring student satisfaction at a UK university. Quality Assurance in Education, 14(3), 251-267. https://doi.org/10.1108/09684880610678568

Gagne, R., \& Driscoll, M. (1988). Essentials of learning for instruction (2nd ed.). Englewood Cliffs, NJ: Prentice Hall. 
Gento Palacios, S., \& Vivas García, M. (2003). El SEUE: un instrumento para conocer la satisfacción de los estudiantes universitarios con su educación. Acción Pedagógica, 12(2).

Gil, J., \& Peña, D. (1998). Un modelo para la determinación de la satisfacción de los estudiantes universitarios con la docencia recibida. VII Congreso Español de Calidad 16-17 junio. Madrid, España.

Hanus, M. D., \& Fox, J. (2015). Assessing the effects of gamification in the classroom: A longitudinal study on intrinsic motivation, social comparison, satisfaction, effort, and academic performance. Computers and Education, 80, 152-161. https://doi.org/10.1016/j.compedu.2014.08.019

Iwamoto, D. H., Taitano, E. J., Hargis, J., \& Vuong, K. (2017). Analyzing the Efficacy of the Testing Effect Using Kahoot on Student Performance. Turkish Online Journal of Distance Education, 18(April), 80-93. Retrieved from https://eric.ed.gov/?id=EJ1145220

Jaber, J. R., Arencibia, A., Carrascosa, C., Ramírez, A. S., Rodríguez-Ponce, E., Melian, C., ... Farray, D. (2016). Empleo de Kahoot como herramienta de gamificación en la docencia universitaria. III Jornadas Iberoamericanas de Innovación Educativa En El Ámbito de Las TIC. Las Palmas de Gran Canaria, España.

Jakubowski, M. (2014). GAMIFICATION IN BUSINESS AND EDUCATION - PROJECT OF GAMIFIED COURSE FOR UNIVERSITY STUDENTS. Developments in Business Simulation and Experiential Learning, 41, 339-342.

Johnson, L., Adams Becker, S., Estrada, V., \& Freeman, A. (2014). NMC Horizon Report: 2014 K-12 Edition. Austin: Texas.

Kaleta, R., \& Joosten, T. (2007). Student response systems: A University of Wisconsin System Study of Clickers. In EDUCAUSE Center for Applied Research (Vol. 2007). Retrieved from http://web2integration.pbworks.com/f/Student+Response+Systems.pdf

Kapp, K. M., Blair, L., \& Rich, M. (2014). The Gamification of Learning and Instruction Fieldbook: Ideas into Practice. Retrieved from https://www.wiley.com/enal/The+Gamification+of+Learning+and+Instruction+Fieldbook\%3A+Ideas+into+Practice-p9781118674437

Kay, R. H., \& LeSage, A. (2009). Examining the benefits and challenges of using audience response systems: A review of the literature. Computers and Education, 53(3), 819-827. https://doi.org/10.1016/j.compedu.2009.05.001

Kebritchi, M., Hirumi, A., \& Bai, H. (2010). The effects of modern mathematics computer games on mathematics achievement and class motivation. Computers and Education, 55(2), 427-443. https://doi.org/10.1016/j.compedu.2010.02.007

Keller, J. M. (1987). Development and use of the ARCS model of instructional design. Journal of Instructional Development, 10(3), 2-10. https://doi.org/10.1007/BF02905780

Kenny, R. F., \& McDaniel, R. (2011). The role teachers' expectations and value assessments of video games play in their adopting and integrating them into their classrooms. British Journal of Educational Technology, 42(2), 197-213. https://doi.org/10.1111/j.1467-8535.2009.01007.x 
Larsen, M. D. (2006). Advice for New and Student Lecturers on Probability and Statistics. Journal of Statistics Education, 14(1). Retrieved from http://jse.amstat.org/v14n1/larsen.html

Lee, J., \& Hammer, J. (2011). Gamification in Education: What, How, Why Bother? Academic Exchange Quarterly, 15(2), 1-5.

Martínez Navarro, G. (2017). Tecnologías y nuevas tendencias en educación: aprender jugando. El caso de Kahoot. Opción: Revista de Ciencias Humanas y Sociales, 33(83), 252-277. Retrieved from https://dialnet.unirioja.es/servlet/articulo?codigo $=6228338$

Mérida Serrano, R., Angulo Romero, J., Jurado Bello, M., \& Diz Pérez, J. (2011). Student training in transversal competences at the University of Cordoba. European Educational Research Journal, 10(1), 34-52. https://doi.org/10.2304/eerj.2011.10.1.34

Molina Álvarez, J. J., Ortiz Colón, A. M., \& Agreda Montoro, M. (2017). Análisis de la integración de procesos gamificados en Educación Primaria. In Innovación docente y uso de las TIC en educación. Retrieved from http://www.enriquesanchezrivas.es/congresotic/archivos/Form_Compet_metodos/Ortiz_Otros_2.pdf

Moreno Martínez, M. N., Leiva Olivencia, J. J., \& Matas Terrón, A. (2016). Mobile learning, Gamificación y Realidad Aumentada para la enseñanza-aprendizaje de idiomas. IJERI: International Journal of Educational Research and Innovation, (6), 16-34. Retrieved from https://www.upo.es/revistas/index.php/IJERI/article/view/1709

Parra Santos, M. T., Molina Jordá, J. M., \& Casanova Pastor, G. (2018). La Aplicación Kahootpara Motivar la Participación Activa en el Aula. In Redes de investigación en docencia universitaria. Retrieved from http://rua.ua.es/dspace/handle/10045/85010\#.XJtggM-Ua94.mendeley

Pérez Juste, R. (2000). La calidad de la educación. In P. Pérez, R.; López, F.; Peralta, M. y Municio (Ed.), Hacia una educación de calidad. Gestión, instrumentos y evaluación. Madrid, España: Narcea Ediciones.

Plump, C. M., \& LaRosa, J. (2017). Using Kahootin the Classroom to Create Engagement and Active Learning: A Game-Based Technology Solution for eLearning Novices. Management Teaching Review, 2(2), 151-158. https://doi.org/10.1177/2379298116689783

Reig, D. (2013). Los significados educativos y sociales de la revolución móvi. Mobile World Capital. Barcelona, ESPAÑA.

Reig, D., \& Vílchez, L. F. (2013). Los jóvenes en la era de la hiperconectividad: tendencias, claves y miradas. Retrieved from www.fundacion.telefonica.com/es/arte_cultura/publicaciones/detalle/182

Roediger III, H. L., Putnam, A. L., \& Smith, M. A. (2011). Ten Benefits of Testing and Their Applications to Educational Practice. Psychology of Learning and Motivation, 55, 1-36. https://doi.org/10.1016/B9780-12-387691-1.00001-6

Rowley, J. (2003). Designing student feedback questionnaires. Quality Assurance in Education, 11(3), 142-149. https://doi.org/10.1108/09684880310488454 
San-Miguel, T., Megías, J., \& Serna, E. (2017). Gamificación en la universidad II: aprendemos a divertirnos enseñando. Se divierten aprendiendo. IN-RED 2017: III Congreso Nacional de Innovación Educativa y Docencia En Red. https://doi.org/10.4995/inred2017.2017.6837

Santana Cabello, R., \& García Juan, L. (2018). La gamificación en la educación superior. Tecnologías emergentes que motivan al estudio y aumentan el rendimiento. In Las competencias y la sociedad del conocimiento (pp. 155-176). Retrieved from www.memoriascimted.com

Simões, J., Redondo, R. D., \& Vilas, A. F. (2013). A social gamification framework for a K-6 learning platform. Computers in Human Behavior, 29(2), 345-353. https://doi.org/10.1016/j.chb.2012.06.007

Smith-Robbins, S. (2011). This Game Sucks: How to Improve the Gamification of Education. Educause Review, 46, 58-59. Retrieved from https://er.educause.edu/articles/2011/2/this-game-sucks-how-toimprove-the-gamification-of-education

Toppino, T. C., \& Gerbier, E. (2014). About practice. Repetition, spacing, and abstraction. Psychology of Learning and Motivation, 60, 113-189. https://doi.org/10.1016/B978-0-12-800090-8.00004-4

Tucker, B. (2012). The Flipped Classroom. Online instruction at home frees class time for learning. Education Next, 12(1), 82-83.

Wang, A. I., \& Lieberoth, A. (2016). The effect of points and audio on concentration, engagement, enjoyment, learning, motivation, and classroom dynamics using KahootIn Thomas Connolly \& Liz Boyle (Eds.), 10th European Conference of Game Based Le (p. 9). Retrieved from http://pure.au.dk/portal/en/publications/the-effect-of-points-and-audio-on-concentration-engagementenjoyment-learning-motivation-and-classroom-dynamics-using-kahoot(f1 ddf129-5e63-4101-80dd1050a162c57d).html 